\title{
RELATIVELY CENTRAL OPERATORS ON ARCHIMEDEAN VECTOR LATTICES II
}

\author{
P. T. N. McPOLIN and A. W. WICKSTEAD
}

(Received 19 July 1983; revised 2 February 1985)

Communicated by G. Brown

\begin{abstract}
We continue the study of operators from an Archimedean vector lattice $E$ into a cofinal sublattice $H$ which have the property that there is $\lambda>0$ such that if $x \in E, h \in H$ and $|x| \leqslant|h|$, then $|T x| \leqslant \lambda|h|$. The collection $Z(E \mid H)$ of all of those operators forms an algebra under composition. We investigate the relationship between the properties of having an identity, being Abelian and being semi-simple for such algebras, culminating in a proof that they are equivalent if $H$ is Dedekind complete. We also study various spectra for such an operator $T$, showing that, apart from 0 , its spectrum relative to $Z(E \mid H)$ is the same as that of $\left.T\right|_{H}$ relative to $Z(H)$ and that of $T$ relative to $\mathscr{L}(E)$ (provided $E$ is a Banach lattice and $H$ is closed).
\end{abstract}

1980 Mathematics subject classification (Amer. Math. Soc.): 47 B 55, 47 A 10, 46 A 40.

\section{Introduction}

In Wickstead (1980) we introduced the class $Z(E \mid H)$ of relatively central operators from an Archimedean vector lattice $E$ into a cofinal sublattice $H$. These are linear operators $T: E \rightarrow H$ with the property that there is a constant $\lambda$ such that if $x \in E, h \in H$ and $|x| \leqslant|h|$, then $|T x| \leqslant \lambda|h|$. If $E=C(\Omega)$ and $H$ is a closed sublattice containing the constants, then a linear operator $T: E \rightarrow H$ is relatively central if and only if it is an averaging operator. Many of the results in Wickstead (1980) were proved by choosing $h \in H_{+}$, restricting $T$ to the ideal $E_{h}$ generated by $h$ in $E$, extending $T$ to the completion of this for the order unit norm induced by $h$, and then applying the Kakutani representation theorem and this fundamental example.

(c) 1986 Australian Mathematical Society $0263-6115 / 86 \$ A 2.00+0.00$ 
$Z(E \mid H)$ need not have a particularly nice order theoretic structure, but if $H$ is a Dedekind complete vector lattice, then so is $Z(E \mid H)$. We also showed in Wickstead (1980) that if $E$ is a Banach lattice and $H$ a closed cofinal sublattice of $E$, then the compact operators in $Z(E \mid H)$ form a lattice. The expression $\|T\|_{n}=\inf \{\lambda \geqslant 0:|T x| \leqslant \lambda|h|$ whenever $x \in E, h \in H$ and $|x| \leqslant|h|\}$ defines a norm on $Z(E \mid H)$ called the natural norm. If $H$ is relatively uniformly complete, then $Z(E \mid H)$ is complete for the natural norm.

In this note we investigate the structure of $Z(E \mid H)$ as an algebra under composition. The natural norm is readily seen to be submultiplicative. It is not entirely clear that an identity for $Z(E \mid H)$ need have norm 1 , but $Z(E \mid H)$ can certainly be renormed as a (real) normed algebra (Rickart (1960)). Examples in Section 3 will show that $Z(E \mid H)$ need not, in general, have an identity, be Abelian nor be semi-simple. It does, however, turn out that there is a very close relationship between these properties. In the case that $H=E$ is a normed lattice, $Z(E \mid H)$ reduces to the familiar class of central operators $Z(E)$ on $E$, which is algebra isomorphic to a dense unital subalgebra of some $C(\Omega)$ (Theorem 1.2 of Wils (1971)), and so is both Abelian and semi-simple. In this case it is well known that the spectrum of $T \in Z(E)$ is the same whether it is taken relative to $Z(E)$ or to the algebra of all bounded linear operators on $E$. In Section 4 we shall see that there are similar results in our context. Before proceeding to the results on the algebra structure of $Z(E \mid H)$, we wish to introduce a restricted class of sublattices which feature in the main results in Section 3.

\section{Projection sublattices}

Throughout this paper, $E$ will denote an Archimedean vector lattice and $H$ a cofinal sublattice of $E$, unless extra conditions are explicitly imposed.

Definition 2.1. (i) A downward directed net $\left\{x_{\gamma}\right\}_{\gamma \in \Gamma}$ in $E_{+}$is said to decrease to 0 relative to $H$ if each $x_{\gamma}-x_{\gamma^{\prime}} \in H$, and if $h \in H_{+}$with $h \leqslant x_{\gamma}$ for each $\gamma \in \Gamma$ implies that $h=0$.

(ii) A positive linear operator $T: E \rightarrow H$ is relatively order continuous (with respect to $H$ ) if for each net $\left\{x_{\gamma}\right\}_{\gamma \in \Gamma}$ in $E_{+}$which decreases to 0 relative to $H$, the net $\left\{T x_{\gamma}\right\}_{\gamma \in \Gamma}$ decrease to 0 in $H$.

(iii) $H$ is a projection sublattice of $E$ if there is a relatively order continuous positive linear projection of $E$ onto $H$.

The following proposition gives a rather more natural criterion for a sublattice to be a projection sublattice. 
Proposition 2.2. A cofinal sublattice $H$ of an Archimedean vector lattice $E$ is a projection sublattice if and only if for each $x \in E_{+}, \inf _{H}\{h \in H: h \geqslant x\}$ and $\sup _{H}\{h \in H: h \leqslant x\}$ both exist and are equal. If $H$ is a projection sublattice of $E$, then the associated relatively order continuous projection is unique and is a lattice homomorphism.

Proof. If $H$ is a projection sublattice, let $P$ denote a projection associated with $H$. If $x \in E_{+}$, then the set $A=\{x-h: h \in H$ and $h \leqslant x\}$ decreases to 0 relative to $H$, so that $\inf _{H}\{P(x)-h: h \in H$ and $h \leqslant x\}=0$. Hence $\sup _{H}\{h \in$ $H: h \leqslant x\}$ exists and is equal to $P(x)$. Similarly $\inf _{H}\{h \in H: h \geqslant x\}$ exists and equals $P(x)$. This establishes both that $\sup _{H}\{h \in H: h \leqslant x\}=\inf _{h}\{h \in H$ : $h \geqslant x\}$, and that $P$ is unique.

Now suppose that $H$ does have the property that for each $x \in E_{+}, \inf _{H}\{h \in H$ : $h \geqslant x\}$ and $\sup _{H}\{h \in H: h \leqslant x\}$ exist and are equal. Define $P: E_{+} \rightarrow H$ by $P(x)=\inf _{H}\{h \in H: h \geqslant x\}$. Then $P$ is clearly positive homogeneous. It is also additive, for if $x, y \in E_{+}$, then we have

$$
\begin{aligned}
P(x+y) & =\sup _{H}\{h \in H: h \leqslant x+y\} \\
& \leqslant \inf _{H}\{h \in H: h \geqslant x\}+\inf _{H}\{h \in H: h \geqslant y\}=P(x)+P(y) \\
& =\sup _{H}\{h \in H: h \leqslant x\}+\sup _{H}\{h \in H: h \leqslant y\} \\
& \leqslant \inf _{H}\{h \in H: h \geqslant x+y\}=P(x+y) .
\end{aligned}
$$

We may now extend $P$ to a positive linear operator from $E$ into $H$ by defining $P(x-y)=P(x)-P(y)$ for $x, y \in E_{+} . P$ is clearly a projection and is a lattice homomorphism since, if $x, y \in E_{+}$, then we have

$$
\begin{aligned}
P(x) \wedge P(y) & =\sup _{H}\{h \in H: h \leqslant x\} \wedge \sup _{H}\{h \in H: h \leqslant y\} \\
& =\sup _{H}\left\{h \wedge h^{\prime}: h, h^{\prime} \in H, h \leqslant x, h^{\prime} \leqslant y\right\} \\
& =\sup _{H}\{h \in H: h \leqslant x \wedge y\}=P(x \wedge y) .
\end{aligned}
$$

All that remains now is to prove that $P$ is relatively order continuous. Suppose that $\left\{x_{\gamma}\right\}_{\gamma \in \Gamma}$ is a net in $E_{+}$which decreases to 0 relative to $H$. Since for each $\gamma$, $\gamma^{\prime} \in \Gamma$, we have $x_{\gamma}-x_{\gamma^{\prime}} \in H$, it follows that $P\left(x_{\gamma}\right)-x_{\gamma}=P\left(x_{\gamma^{\prime}}\right)-x_{\gamma^{\prime}}$. If $h \in H_{+}$with $h \leqslant P\left(x_{\gamma}\right)$ for all $\gamma \in \Gamma$, we must show that $h=0$. Fix $\gamma_{0} \in \Gamma$ and observe that

$$
h \leqslant x_{\gamma}+P\left(x_{\gamma}\right)-x_{\gamma}=x_{\gamma}+\left[P\left(x_{\gamma_{0}}\right)-x_{\gamma_{0}}\right] \quad(\gamma \in \Gamma) .
$$

Thus if $h^{\prime} \geqslant\left[P\left(x_{\gamma_{0}}\right)-x_{\gamma_{0}}\right]^{+}$we have $h-h^{\prime} \leqslant x_{\gamma}$ for all $\gamma \in \Gamma$, so that $h \leqslant h^{\prime}$. Thus

$$
\begin{aligned}
h & \leqslant \inf _{H}\left\{h^{\prime} \in H: h^{\prime} \geqslant\left[P\left(x_{\gamma_{0}}\right)-x_{\gamma_{0}}\right]^{+}\right\} \\
& =\sup _{H}\left\{h^{\prime} \in H: h^{\prime} \leqslant\left[P\left(x_{\gamma_{0}}\right)-x_{\gamma_{0}}\right]^{+}\right\} \\
& =0,
\end{aligned}
$$


since $h^{\prime} \leqslant\left[P\left(x_{\gamma_{0}}\right)-x_{\gamma_{0}}\right]^{+}$implies that

$$
\begin{aligned}
h^{\prime} & =P\left(h^{\prime}\right) \leqslant P\left(\left[P\left(x_{\gamma_{0}}\right)-x_{\gamma_{0}}\right]^{+}\right) \\
& =\left(P\left[P\left(x_{\gamma_{0}}\right)-x_{\gamma_{0}}\right]\right)^{+} \\
& =0 .
\end{aligned}
$$

ExAmple 2.3. If $E=\mathbb{R}^{2}$ with the usual order, and if $H=\{(x, x): x \in \mathbb{R}\}$, then there are many positive projections of $E$ onto $H$, but $H$ is not a projection sublattice of $E$ as

$$
\inf _{H}\{h \in H: h \geqslant(0,1)\}=(1,1) \neq(0,0)=\sup _{H}\{h \in H: h \leqslant(0,1)\} .
$$

In view of the importance of our basic example of $E=C(\Omega)$ and $H$ a closed sublattice containing the constants, it is of obvious interest to know as much as possible about the projection sublattices in this case. Whilst we do not have a complete description, we do have sufficiently many results to construct a significant example. If $P$ is a lattice homomorphism, and a projection, of $E$ onto $H$, then there is a continuous map $\phi: \Omega \rightarrow \Omega$ such that $P f=f \circ \phi$ (Ellis (1964)). The fact that $P$ is a projection implies that $\phi$ is a retract of $\Omega$ onto $K=\phi(\Omega)$. Let $\tau$ denote the topology of $\Omega$ relativised to $K$. The set $\phi(\Omega \backslash K)$ is contained in $K$. Let $Q$ denote its interior for the $\tau$ topology.

\section{EXAMPLE 2.4. With the notation above:}

(i) If $Q=\varnothing$, then $H$ is a projection sublattice.

(ii) If $H$ is a projection sublattice and $K$ is $a G_{\delta}$ in $\Omega$, then $Q=\varnothing$.

Proof. (i) If $f \in E^{+}$, let $f^{\uparrow}: K \rightarrow \mathbb{R}$ be defined by $f^{\uparrow}(y)=\sup \{f(x)$ : $\phi(x)=y\}$, so that $f^{\uparrow}$ is upper semi-continuous by, for example, 7.5.5 of Semadeni (1971). If $A=\{h \in H: h \geqslant f\}$, then $A=\left\{g \circ \phi: g \in E\right.$ and $\left.g \circ \phi\right|_{K}$ $\left.\geqslant f^{\uparrow}\right\}$. As $f^{\uparrow}$ is u.s.c., for each $y \in K$ we have $f^{\uparrow}(y)=\inf \{g(y): g \in C(K)$ and $\left.g \in f^{\uparrow}\right\}$, by 6.3 .4 of Semadeni (1971). Hence $f^{\uparrow}(y)=\inf \{g \circ \phi(y): g \circ \phi$ $\in A\}$, since $\left\{\left.g \circ \phi\right|_{K}: g \in C(\Omega)\right\}=C(K)$ by the Tietze-Urysohn extension theorem and the fact that $\phi^{2}=\phi$. Thus if $h$ is any lower bound in $H$ for $A$, we must have $\left.h\right|_{K} \leqslant f^{\uparrow}$. However, $\left.f^{\uparrow}\right|_{K \backslash \phi(\Omega \backslash K)}=\left.f\right|_{K \backslash \phi(\Omega \backslash K)}$, so that $\left.h\right|_{k \backslash \phi(\Omega \backslash K)} \leqslant$ $\left.f\right|_{K \backslash \phi(\Omega \backslash K)}$. If $Q=\varnothing$, then $K \backslash \phi(\Omega \backslash K)$ is dense in $K$, so that, by continuity, $\left.h\right|_{K} \leqslant\left. f\right|_{K}$. Hence $h=h \circ \phi \leqslant f \circ \phi$, and it follows that $\inf _{H}\{h \in H: h \geqslant f\}=$ $f \circ \phi$. The proof that $\sup _{H}\{h \in H: h \leqslant f\}=f \circ \phi$ is similar, and so $H$ must be a projection sublattice of $E$.

(ii) Observe first that Proposition 2.2 tells us that $\inf _{H}\{h \in H: h \geqslant f\}=$ $\sup _{H}\{h \in H: h \leqslant f\}=P f=f \circ \phi$. As we are supposing $\Omega \backslash K$ to be an $F_{o}$ in $\Omega$, we have $\Omega \backslash K=\bigcup_{n=1}^{\infty} F_{n}$, where each $F_{n}$ is closed in $\Omega$. As $\phi(\Omega \backslash K)=\bigcup_{n=1}^{\infty}\left(F_{n}\right)$, if we had $Q \neq \varnothing$, then for some $n \in \mathbb{N}, \phi\left(F_{n}\right)$ would have a non-empty 
$\tau$-interior, since $K$ is a Baire space. Let $x_{0} \in F_{n}$ with $\phi\left(x_{0}\right)$ in the $\tau$-interior of $\phi\left(F_{n}\right)$. There is $f \in C(\Omega)_{+}$with $\left.f\right|_{F_{n}} \equiv 1$ and $f\left(\phi x_{0}\right)=0$. Now $\inf \{h \in H$ : $h \geqslant f\} \neq f \circ \phi$, for if we define $h_{0} \in C(K)$, so that $0 \leqslant h_{0} \leqslant 1, h_{0}\left(\phi x_{0}\right)=1$, and $h_{0}$ vanishes off the $\tau$-interior of $\phi\left(F_{n}\right)$, and if we let $\bar{h}_{0}$ be any norm-preserving extension of $h_{0}$ to $C(\Omega)$, then we have $\left.\bar{h}_{0} \circ \phi\right|_{K} \leqslant f^{\uparrow}$, and yet $\bar{h}_{0} \circ \phi \nless f \circ \phi$.

Note 2.5. If, in fact, $Q=\varnothing$ and $K$ is a $G_{\delta}$ in $\Omega$, then for each $f \in C(\Omega)_{+}$, there are countable sets $H_{1}$ and $H_{2}$, with $h_{1} \leqslant f \leqslant h_{2}$ for each $h_{1} \in H_{1}$ and $h_{2} \in H_{2}$, such that $\inf _{H} H_{2}=\sup _{H} H_{1}$.

EXAMPLE 2.6. Let $\Omega$ be any Stonean space, and let $\omega_{0}$ be a non-isolated point in $\Omega$, (e.g. $\Omega=\beta(\mathbb{N})$ ) and $\omega_{0} \in \beta(\mathbb{N} \backslash \mathbb{N})$. Let $\infty$ be some object not in $\Omega$ and give $X=\Omega \cup\{\infty\}$ the disjoint union topology, under which it is a Stonean space. Let $\phi: X \rightarrow X$ be defined by $\left.\phi\right|_{\Omega}=\mathrm{id}_{\Omega}$ and $\phi(\infty)=\omega_{0}$. Then $\{f \circ \phi: f \in C(X)\}=$ $\left\{f \in C(X): f(\infty)=f\left(\omega_{0}\right)\right\}$ is a proper Dedekind complete projection sublattice of $C(X)$, since $\phi(X \backslash K)=\phi(\{\infty\})=\left\{\omega_{0}\right\}$ has empty $\tau$-interior as $\omega_{0}$ is not isolated in $K=\Omega$.

Some idea of the importance of projection sublattices in the theory of relatively central operators is given by:

PROPOSITION 2.7. If $E$ is an Archimedean vector lattice and $H$ a cofinal projection sublattice of it then the restriction map from $Z(E \mid H)$ into $Z(H)$ is an order and algebra isomorphism.

Proof. It is immediate from Proposition 2.2 that the relatively order continuous projection $P$ from $E$ onto $H$ lies in $Z(E \mid H)$. If $S \in Z(E \mid H)$ and $x \in E_{+}$, then $|S x| \leqslant\|S\|_{n} h$ for each $h \in H$ with $x \leqslant h$, so that $|S x| \leqslant\|S\|_{n} \cdot P x$. Thus $P$ is an order unit for $Z(E \mid H)$, and in particular $Z(E \mid H)$ is positively generated. We show that for each $T \in Z(E \mid H)$, we have $T \circ P=T$. In order to prove this it clearly suffices to prove that if $T \in Z(E \mid H)_{+}$and $x \in E_{+}$, then $T(P x)=T x$. But if $h \in H$ with $h \geqslant x$, we have $T h \geqslant T x$ and, as $\left.T\right|_{H} \in Z(H)$ is order continuous,

$$
\begin{aligned}
T(P x) & =T\left(\inf _{H}\{h: h \geqslant x, h \in H\}\right) \\
& =\inf _{H}\{T h: h \geqslant x, h \in H\} \geqslant T x .
\end{aligned}
$$

We see similarly that $T(P x) \leqslant T x$, thus establishing that $T \circ P=T$. 
It follows immediately that $\left.T \rightarrow T\right|_{H}$ is injective, since $T \circ P=\left(\left.T\right|_{H}\right) \circ P$. The map $\left.T \rightarrow T\right|_{H}$ is surjective, since if $T \in Z(H)$, then $T \circ P \in Z(E \mid H)$ and $\left.T \circ P\right|_{H}=T$. The map is clearly positive, and if $\left.T\right|_{H} \geqslant 0$, then $T=\left(\left.T\right|_{H}\right) \circ P \geqslant 0$, so that the order isomorphism is established. That the restriction map is an algebra homomorphism is clear, and so the proof is complete.

In particular, note that Proposition 2.7 together with Example 2.6 tells us that we cannot hope to deduce that $H=E$ from properties of $Z(E \mid H)$.

\section{The algebra structure of $Z(E \mid H)$}

The first task we shall devote ourselves to in this section is to determine the relationship between the properties of having an identity, being Abelian, and being semi-simple for $Z(E \mid H)$. Let us first notice that we have easily:

LEMMA 3.1. If $E$ is an Archimedean vector lattice, if $H$ is a cofinal sublattice of $E$, and if $Z(E \mid H)$ has an identity, then $Z(E \mid H)$ is isomorphic to a subalgebra of $Z(H)$, and so is Abelian and semi-simple.

Proof. If $J$ is an identity for $Z(E \mid H)$, then the restriction map $\left.T \rightarrow T\right|_{H}$, which is an algebra homomorphism, is injective, since $T=T \circ J=\left(\left.T\right|_{H}\right) \circ J$. Hence $T=0$ if and only if $\left.T\right|_{H}=0$.

The converse of this is false.

EXAMPLE 3.2. In Example 4.3(ii) of Wickstead (1980) we saw that $Z\left(l_{\infty}, c\right)$ may be identified with $c_{0}$ for both the order and algebraic structures. This is semisimple and Abelian but has no identity.

Problem 3.3. If $Z(E \mid H)$ has an identity, must the restriction to $Z(H)$ be bipositive? In particular, it would follow that the identity would be an order unit. This is the case if $Z(E \mid H)$ is positively generated.

The properties of being Abelian and semisimple are equivalent for $Z(E \mid H)$, provided that $Z(E \mid H)$ is positively generated, but before we prove this we shall identify the radical $\mathscr{R}(Z(E \mid H))$ of $Z(E \mid H)$.

Proposition 3.4. If $E$ is an Archimedean vector lattice, $H$ a cofinal sublattice of $E$, and $T \in Z(E \mid H)$, then

$$
\nu(T) \underset{\text { def }}{=} \inf \left\{\left\|T^{k}\right\|_{n}^{1 / k}: k \in \mathbb{N}\right\}=\left\|\left.T\right|_{H}\right\|_{n} .
$$


Proof.

$$
\begin{aligned}
\nu(T) & =\inf \left\{\left\|\left(\left.T\right|_{H}\right)^{k-1} T\right\|_{n}^{1 / k}: k \in \mathbb{N}\right\} \\
& \leqslant \inf \left\{\left\|\left(\left.T\right|_{H}\right)^{k-1}\right\|_{n}^{1 / k} \cdot\|T\|_{n}^{1 / k}: k \in \mathbb{N}\right\} \\
& =\inf \left\{\left(\left\|\left.T\right|_{H}\right\|_{n}^{k-1}\right)^{1 / k}\|T\|_{n}^{1 / k}: k \in \mathbb{N}\right\} \\
& =\left\|\left.T\right|_{H}\right\|_{n} .
\end{aligned}
$$

Conversely, the fact that $\left\|T^{k}\right\|_{n} \geqslant\left\|\left.T^{k}\right|_{H}\right\|$ for each $k \in \mathbb{N}$ shows that $\nu(T) \geqslant$ $\nu\left(\left.T\right|_{H}\right)=\left\|\left.T\right|_{H}\right\|_{n}$.

COROllary 3.5. If $E$ is an Archimedean vector lattice and $H$ a cofinal sublattice of $E$, then

$$
\mathscr{R}(Z(E \mid H))=\{T \in Z(E \mid H): \nu(T)=0\}=\left\{T \in Z(E \mid H):\left.T\right|_{H}=0\right\} .
$$

Proof. By Proposition 3.4, the last two sets coincide. The set $\{T \in Z(E \mid H)$ : $\left.\left.T\right|_{H}=0\right\}$ is a two-sided ideal in $Z(E \mid H)$ consisting of nilpotent elements (as each such $T$ has zero square), so it is contained in $\mathscr{R}(Z(E \mid H))$ by Rickart (1960), Theorem 2.3.2. On the other hand, Theorem 2.3 .4 of Rickart (1960) tells us that $\mathscr{R}(Z(E \mid H))$ is contained in $\{T \in Z(E \mid H): \nu(T)=0\}$, so the equality of all three sets is established.

Proposition 3.6. If $E$ is an Archimedean vector lattice and $H$ a cofinal sublattice of $E$, then

(i) if $Z(E \mid H)$ is semi-simple, then $Z(E \mid H)$ is Abelian;

(ii) if $Z(E \mid H)$ is Abelian and positively generated, then $Z(E \mid H)$ is semi-simple.

Proof. (i) If $S, T \in Z(E \mid H)$, then $\left.S T\right|_{H}=\left.\left.S\right|_{H} T\right|_{H}=\left.\left.T\right|_{H} S\right|_{H}$, so that $\left.(S T-T S)\right|_{H}=0$. Hence $S T-T S \in \mathscr{R}(Z(E \mid H))=\{0\}$, so that $S T=T S$.

(ii) Suppose $\left.T\right|_{H}=0$. Then for every $h \in H_{+},\left.T\right|_{H_{h}}=0$. If we can show that this forces $\left.T\right|_{E_{h}}=0$, then we have $T=0$, and $Z(E \mid H)$ is semi-simple. This argument, together with a uniform extension process, as indicated at the top of page 194 of Wickstead (1980), allows us to consider our basic example (Wickstead (1980), Theorem 2.3).

Let $S, T \in Z(C(\Omega) \mid C(\pi) C(\Sigma))$ be represented by the mappings $\lambda$ and $\mu$, respectively, and suppose that $S \geqslant T,-T$. For $\sigma \in \Sigma$ and $f \in C(\Omega)$, we have

$$
\begin{aligned}
\int\left(\int f d \mu(\sigma)\right) d \lambda(\sigma) & =\int(T f) d \lambda(\sigma)=\left.S T(f)\right|_{\pi^{-1} \sigma} \\
& =\left.T S(f)\right|_{\pi^{-1} \sigma}=0,
\end{aligned}
$$


since $S f \in C(\pi) C(\Sigma)$ and $\left.T\right|_{C(\pi) C(\Sigma)}=0$. Thus, either $\mu(\sigma)=0$ or else $\int 1 d \lambda(\sigma)$ $=0$. As $\lambda(\sigma) \geqslant 0$, this forces $\lambda(\sigma)=0$, and hence $\mu(\sigma)=0$, since $\lambda(\sigma) \geqslant|\mu(\sigma)|$. Thus, in either case, $\mu(0)=0$, and hence $T=0$.

Problem 3.7. Does $Z(E \mid H)$ Abelian always imply that $Z(E \mid H)$ is semisimple?

It is not the case that $Z(E \mid H)$ is always Abelian.

EXAMPLE 3.8. Let $E=\mathbb{R}^{2}$, and let $H=\{(x, x): x \in \mathbb{R}\}$ with the usual order. Then $Z(E \mid H)$ may be identified with $\mathbb{R}^{2}$ via the correspondence $T \leftrightarrow(a, b)$, where

$$
T(x, y)=(a x+b y, a x+b y) .
$$

If we define $R, S \in Z(E \mid H)$ by $R(x, y)=(x, x)$ and $S(x, y)=(y, y)$, then $R S=S \neq R=S R$, so that $Z(E \mid H)$ is not Abelian (and hence certainly not semi-simple).

If $H$ is Dedekind complete, the relationship between the various algebraic properties that $Z(E \mid H)$ may have is much clearer.

THEOREM 3.9. If $E$ ia an Archimedean vector lattice and $H$ a Dedekind complete cofinal sublattice of $E$, then the following are equivalent.

(a) $Z(E \mid H)$ has an identity.

(b) $Z(E \mid H)$ is Abelian.

(c) $Z(E \mid H)$ is semi-simple.

(d) The restriction map of $Z(E \mid H)$ into $Z(H)$ is a surjective lattice and algebra isomorphism.

(e) $H$ is a projection sublattice of $E$.

Proof. Proposition 2.7 asserts precisely that (e) $\Rightarrow(d)$. Clearly (d) $\Rightarrow$ (a) and (a) $\Rightarrow$ (c) by Lemma 3.1. The fact that (c) $\Rightarrow$ (b) constitutes Proposition 3.6(i), and so we need only prove that $(b) \Rightarrow(e)$.

Suppose $H$ is not a projection sublattice of $E$. Then we shall prove that $Z(E \mid H)$ is not Abelian. In this case Proposition 2.2 tells us that we can find $x \in E_{+}$with

$$
h_{1}=\inf \{h \in H: h \geqslant x\} \neq \sup \{h \in H: h \leqslant x\}=h_{2} .
$$

If $x_{1}=h_{1}-x$ and $x_{2}=x-h_{2}$, then $x_{1}$ and $x_{2}$ must be linearly independent, and $x_{1}+x_{2}=h_{1}-h_{2} \in H_{+} \backslash\{0\}$. If $K$ is the linear span of $x_{1}$ and $x_{2}$, then we may define linear maps $T_{1}, T_{2}: K \rightarrow\left(x_{1}+x_{2}\right) \cdot \mathbb{R}$ by $T_{1}\left(\lambda x_{1}+\mu x_{2}\right)=\lambda\left(x_{1}+\right.$ $\left.x_{2}\right)$ and $T_{2}\left(\lambda x_{1}+\mu x_{2}\right)=\mu\left(x_{1}+x_{2}\right)$. Once we have shown that $T_{1}$ and $T_{2}$ may be extended to $\bar{T}_{1}, \bar{T}_{2} \in Z(E \mid H)$, the proof will be complete, since $T_{1}$ and $T_{2}$ do not commute, and so their extensions certainly cannot. 
Define a sublinear map $p: E \rightarrow H$ by $p(x)=\inf _{H}\{h \in H: h \geqslant|x|\}$. If we show that $h \in H$, and $h \geqslant\left|\lambda x_{1}+\mu x_{2}\right|$ imply that $h \geqslant \max \{|\lambda|,|\mu|\}\left(x_{1}+x_{2}\right)$, then it will follows that $\left|T_{i}(y)\right| \leqslant p(y)$ for $i=1$, 2. If $\lambda=\mu$ there is nothing to prove. We now consider separately the cases $\lambda>\mu$ and $\lambda<\mu$.

If $\lambda>\mu$, then $h \geqslant\left|\lambda x_{1}+\mu x_{2}\right|$ implies that $h \geqslant \lambda x_{1}+\mu x_{2}=(\lambda-\mu) x_{1}+$ $\mu\left(x_{1}+x_{2}\right)$, so that $(\lambda-\mu) x_{1} \leqslant h-\mu\left(x_{1}+x_{2}\right)=h^{\prime} \in H$. Thus

$$
(\lambda-\mu)^{-1} h^{\prime} \geqslant x_{1}=h_{1}-x,
$$

which implies that

$$
h_{1}-(\lambda-\mu)^{-1} h^{\prime} \leqslant x .
$$

As $h_{2}=\sup \{g \in H: g \leqslant x\}$, it follows that $h_{1}-(\lambda-\mu)^{-1} h^{\prime} \leqslant h_{2}$. Hence

$$
\begin{aligned}
h & =\mu\left(x_{1}+x_{2}\right)+h^{\prime} \geqslant \mu\left(x_{1}+x_{2}\right)+(\lambda-\mu)\left(h_{1}-h_{2}\right) \\
& =\lambda\left(x_{1}+x_{2}\right) .
\end{aligned}
$$

Similarly, if $\lambda<\mu$, we can show that $h \geqslant \mu\left(x_{1}+x_{2}\right)$, so that $h \geqslant \max \{\lambda, \mu\}\left(x_{1}\right.$ $\left.+x_{2}\right)$. The same argument with $\lambda$ replaced by $-\lambda$ and $\mu$ by $-\mu$ will show that $h \geqslant \max \{-\lambda,-\mu\}\left(x_{1}+x_{2}\right)$, so that $h \geqslant \max \{|\lambda|,|\mu|\}\left(x_{1}+x_{2}\right)$.

We may now use the Hahn-Banach extension theorem to extend $T_{1}$ and $T_{2}$ to $\bar{T}_{1}$ and $\bar{T}_{2}$ defined on the whole of $E$ and dominated by $p$. These extensions are clearly relatively central, and so the proof is complete.

We conclude this section by pointing out a useful property that $Z(E \mid H)$ must have if $H$ is uniformly complete. This is of special interest in view of Example 3.10(ii) of Huijsmans and De Pagter (1982), which exhibits a uniformly complete $f$-algebra in which square roots do not exist.

Theorem 3.10. Let $E$ be an Archimedean vector lattice and $H$ a uniformly complete cofinal sublattice of $E$. If $T \in Z(E \mid H)_{+}$and $n \in \mathbb{N}$, then $T$ has a unique positive n'th root $S \in Z(E \mid H)$ with $\|S\|_{n}=\|T\|_{n}^{1 / n}$.

Proof. Consider first the basic example. Let $\mu$ represent $T \in$ $Z(C(\Omega) \mid C(\pi) C(\Sigma)$ ), and let $f=T 1$. Note that if $g \in C(\Omega)$, then

$$
\begin{aligned}
|T g(\sigma)| & =\left|\int g d \mu(\sigma)\right| \leqslant\|g\|_{\infty}\left|\int 1 d \mu(\sigma)\right| \\
& =\|g\|_{\infty}|f(\sigma)| .
\end{aligned}
$$

Thus if $f(\sigma)>0$, we have

$$
\left|T g(\sigma) / f(\sigma)^{(n-1) / n}\right| \leqslant\|g\|_{\infty} f(\sigma)^{1 / n} .
$$


The left hand function in this inequality is continuous on $\{\sigma \in \Sigma: f(\sigma)>0\}$ and will extend continuously to the whole of $\Sigma$ by defining it to be zero on $\{\sigma \in \Sigma$ : $f(\sigma)=0\}$. Call this extension $S g(\sigma)$. The map $g \rightarrow S g$ is clearly a positive linear map of $C(\Omega)$ into $C(\pi) C(\Sigma)$. It is relatively central because

$$
S^{*}\left(\varepsilon_{g}\right)=f(\sigma)^{-(n-1) / n} \nu(\sigma),
$$

and because of Theorem 2.3 of Wickstead (1980). Note that

$$
S^{n} g=\left(\left.S\right|_{H}\right)^{n-1} S g=\left[f \cdot f^{-(n-1) / n}\right]^{(n-1)}\left[f^{-(n-1) / n}\right] T g=T g .
$$

This mapping $S$ is the only possible positive $n$th root of $T$, for if $U^{n}=T, U \geqslant 0$, and if $U$ is represented by $\nu$, then the uniqueness is clear on $\{\sigma \in \Sigma: f(\sigma)>0\}$. If $f(\sigma)=0$, then $\mu(\sigma)=0$ (as $\mu \geqslant 0$ ), so that $\left[\int 1 d \nu(\sigma)\right]^{n-1} \nu(\sigma)=0$. The positivity of $\nu$ implies that $\nu(\sigma)=0$. The equality for the norm is obvious.

Consider now the general case. If $h \in H_{+}$, consider $\left.T\right|_{E_{h}}$. This may be extended, by continuity for the natural norm, to $\hat{T} \in Z\left(\hat{E}_{h} \mid E_{h}\right)$, where $\hat{E}_{h}$ is the completion of $\hat{E}_{h}$ for the natural norm. By the basic example, and by the Kakutani representation, $T$ has a unique positive $n$th root $S \in Z\left(\hat{E}_{h} \mid H_{h}\right)$. Now $\left.S\right|_{F_{h}}$ is the unique positive $n$th root of $\left.T\right|_{E_{h}}$. The uniqueness allows us to piece together these operators as $h$ varies, so as to obtain the unique positive $n$th root of $T$. The relationship between the norms follows easily once we note that, for $U \in Z(E \mid H)$, we have

$$
\|U\|_{n}=\sup \left\{\left\|\left.U\right|_{E_{h}}\right\|_{n}: h \in H_{+}\right\}
$$

\section{Spectra for relatively central operators}

If $T$ is a central operator on a Banach lattice $E$, then we may calculate the two spectra $\sigma_{Z(E)}(T)$ and $\sigma_{\mathscr{L}\left(E_{\mathrm{c}}\right)}(T)$ relative to the algebras $Z(E)$ and $\mathscr{L}\left(E_{\mathrm{c}}\right)$ (the algebra of all bounded linear operators on the complexification of $E$ ) respectively. It is well known that the two spectra coincide. When dealing with relatively central operators from $E$ into $H$, we have $\sigma_{Z(H)}\left(\left.T\right|_{H}\right)$ available as well as $\sigma_{Z(E \mid H)}(T)$, which replaces $\sigma_{Z(E)}(T)$. Proposition 3.4 and Corollary 3.5 lead us to expect these to be closely related in general, even if $E$ is not a Banach lattice. This is indeed the case:

Proposition 4.1. If $E$ is an Archimedean vector lattice and $H$ a cofinal sublattice of $E$, then for each $T \in Z(E \mid H)$, we have

$$
\sigma_{Z(E \mid H)}(T) \backslash\{0\}=\sigma_{Z(H)}\left(\left.T\right|_{H}\right) \backslash\{0\} .
$$


Proof. If $0 \neq \lambda \in \sigma_{Z(H)}\left(\left.T\right|_{H}\right)$, then $\left(\left.\lambda^{-1} T\right|_{H}\right)$ is quasi-singular. This means that for each $U \in Z(H)$, either $U \circ\left(\left.\lambda^{-1} T\right|_{H}\right)$ or $\left(\left.\lambda^{-1} T\right|_{H}\right) \circ U$ is non-zero, where $V \circ W=V+W-V W$ is the circle operation defined on page 16 of Rickart (1960). In particular, if $S \in Z(E \mid H)$, then either $\left(\left.S\right|_{H}\right) \circ\left(\left.\lambda^{-1} T\right|_{H}\right)$ or $\left(\left.\lambda^{-1} T\right|_{H}\right) \circ\left(\left.S\right|_{H}\right)$ is non-zero. Hence, one of $S \circ\left(\lambda^{-1} T\right)$ and $\left(\lambda^{-1} T\right) \circ S$ is non-singular, since $\left.\left.V\right|_{H} \circ W\right|_{H}=\left.(V \circ W)\right|_{H}$. Hence $\lambda^{-1} T$ is quasi-singular in $Z(E \mid H)$ and $\lambda \in \sigma_{Z(E \mid H)}(T)$.

If we now have $0 \neq \lambda \notin \sigma_{Z(H)}\left(\left.T\right|_{H}\right)$, then $\left.(\lambda-T)\right|_{H}$ has an inverse in $Z(H)$. If $S=-\left(\lambda-\left.T\right|_{H}\right)^{-1} T$, then routine calculations show that $S \circ\left(\lambda^{-1} T\right)=$ $\left(\lambda^{-1} T\right) \circ S=0$, so that $\lambda \notin \sigma_{Z(E \mid H)}(T)$.

Notes 4.2. (i) Examples 4.5(i) and (ii) will show that the exclusion of 0 is essential.

(ii) In particular, this tells us that $\sigma_{Z(E \mid H)}(T) \subseteq \mathbb{R}$.

(iii) The proof shows that $\sigma_{Z(E \mid H)}(T) \backslash\{0\}=\sigma_{Z(H)}\left(\left.T\right|_{H}\right) \backslash\{0\}$ if $E$ is normed and $T \in Z(E \mid H)$, the continuous operators in $Z(E \mid H)$.

THEOREM 4.3. If $E$ is a Banach lattice and $H$ a closed sublattice of $E$ such that $E_{H}$, the ideal generated by $H$ in $E$, is dense in $E$, then for each $T \in Z(E \mid H)$, we have

$$
\sigma_{\mathscr{L}\left(E_{\mathrm{c}}\right)}(T) \backslash\{0\}=\sigma_{Z(E \mid H)}(T) \backslash\{0\}
$$

Proof. Recall that if $0 \neq \lambda \in \sigma_{Z(E \mid H)}(T)$, then $\lambda \in \sigma_{Z(H)}\left(\left.T\right|_{H}\right)$ by Proposition 4.1 (we may identify $Z\left(E_{H} \mid H\right)$ with $Z(E \mid H)$ by continuity). As the spectrum is all boundary, $\lambda$ lies in the approximate point spectrum of $\left.T\right|_{H_{\mathrm{c}}}$ (Schaefer (1974), page 310 ). Clearly $\lambda$ will also lie in the approximate point spectrum of $T$, and hence $\lambda \in \sigma_{\mathscr{L}\left(E_{\mathrm{c}}\right)}(T)$.

Now suppose that $\lambda \neq 0$ and that $\lambda$ lies in the approximate point spectrum of $T$. Choose $x_{i} \in E$ with $\left\|x_{i}\right\|=1$ and $\left\|(T-\lambda) x_{i}\right\| \rightarrow 0$. Then

$$
(T-\lambda)\left(T x_{i}\right)=T\left((T-\lambda) x_{i}\right) \rightarrow 0,
$$

but

$$
\left\|T x_{i}\right\|=\left\|(T-\lambda) x_{i}+\lambda x_{i}\right\| \rightarrow|\lambda| \neq 0,
$$

so that (recalling that $T x_{i} \in H$ ), $\lambda$ lies in the approximate point spectrum of $\left.T\right|_{H_{\mathrm{c}}}$. Thus the non-zero boundary points of $\sigma_{\mathscr{L}\left(E_{\mathrm{c}}\right)}(T)$ lie in $\sigma_{\mathscr{L}(H)}\left(\left.T\right|_{H_{\mathrm{c}}}\right)=$ $\sigma_{Z(H)}\left(\left.T\right|_{H}\right)$. But $\sigma_{Z(H)}\left(\left.T\right|_{H}\right) \subseteq \mathbf{R}$, so that $\sigma_{\mathscr{L}_{\left(E_{\mathrm{C}}\right)}}(T)$ is all boundary and we certainly have

$$
\sigma_{\mathscr{L}\left(E_{\mathrm{C}}\right)}(T) \subset \sigma_{Z(H)}\left(\left.T\right|_{H}\right) \cup\{0\} .
$$

Combining this with Proposition 4.1 gives the desired inclusion. 
We conclude the paper with a discussion of zero.

LEMMA 4.4. With the notation of Theorem 4.3, if $0 \in \sigma_{Z(E \mid H)}(T)$, or if $0 \in$ $\sigma_{Z(H)}\left(\left.T\right|_{H}\right)$, then $0 \in \sigma_{\mathscr{L}\left(E_{\mathbf{c}}\right)}(T)$.

Proof. If $0 \notin \sigma_{\mathscr{L}\left(E_{\mathrm{c}}\right)}(T)$, then $T$ is invertible, and so in particular is surjective. It follows that $H=E$, so that $Z(E \mid H)=Z(H)=Z(E)$, and we are reduced to the well-known equality $\sigma_{\left.\mathscr{L}_{(} E_{\mathrm{c}}\right)}(T)=\sigma_{Z(E)}(T)$ for central operators $T$.

The following examples show that these are the only implications that do hold for zero.

ExAMPLes 4.5. (i) Recall from Example 2.6(iii) of Wickstead (1980) that there is a Banach lattice $E$ and a closed cofinal sublattice $H$ of $E$ with $Z(E \mid H)=\{0\}$. If $\Omega$ is any compact Hausdorff space, then we may identify $Z(C(\Omega) \oplus E \mid C(\Omega) \oplus H)$ with $Z(C(\Omega))$. The band projection $P$ of $C(\Omega) \oplus E$ onto $C(\Omega) \oplus\{0\}$ is the identity of this algebra, and so $0 \notin \sigma_{Z(C(\Omega) \oplus E \mid C(\Omega) \oplus H)}(P)$. Both $P$ and $\left.P\right|_{C(\Omega) \oplus H}$ are proper projections, so that $0 \in \sigma_{\mathscr{L}(C(\Omega) \oplus E)}(P)$, and $0 \in \sigma_{Z(C(\Omega) \oplus H)}\left(\left.P\right|_{C(\Omega) \oplus H}\right)$.

(ii) In Example 3.8, $Z(E \mid H)$ has no identity, for otherwise it would be Abelian. Thus $0 \in \sigma_{Z(E \mid H)}(R)$. Also $R$ is not surjective, whence $0 \in \sigma_{\mathscr{L}_{\left(E_{\mathrm{C}}\right)}}(R)$. On the other hand, $\left.R\right|_{H}$ is the identity on $H$, so that $0 \notin \sigma_{Z(H)}\left(\left.R\right|_{H}\right)$.

\section{References}

A. J. Ellis (1964), 'Extreme positive operators', Quart. J. Math. (2) 15, 342-344.

C. B. Huijsmans and B. De Pagter (1982), 'Ideal theory in f-algebras', Trans. Amer. Math. Soc. 269, 225-245.

C. E. Rickart (1960), General theory of Banach algebras (Van Nostrand, Princeton).

H. H. Schaefer (1974), Banach lattices and positive operators (Springer-Verlag, Berlin, Heidelberg, New York).

Z. Semadeni (1971), Banach spaces of continuous functions (Polish Scientific Publishers, Warsaw).

A. W. Wickstead (1980), 'Relatively central operators on Archimedean vector lattices-I', Proc. Royal Irish Acad. Sect. A 80, 191-208.

W. Wils (1971), 'The ideal center of partially ordered vector spaces', Acta Math. 127, 41-77.

St. Joseph's College of Education

Belfast BT11 9GA

Northern Ireland
Department of Pure Mathematics

The Queen's University of Belfast

Belfast BT7 INN

Northern Ireland 